\title{
How to manage continuing education and retraining programs on optical physics and laser technology at a university: Moscow State experience
}

\section{Victor Zadkov, Nikolai Koroteev}

Victor N. Zadkov, Nikolai I. Koroteev, "How to manage continuing education and retraining programs on optical physics and laser technology at a university: Moscow State experience," Proc. SPIE 2525, 1995 International Conference on Education in Optics, (13 October 1995); doi: $10.1117 / 12.224080$

Event: SPIE's 1995 International Symposium on Optical Science, Engineering, and Instrumentation, 1995, San Diego, CA, United States 
How to Manage Continuing Education and Retraining Programs on Optical Physics and Laser Technology at a University: Moscow State Experience

\author{
Victor Zadkov, Nikolai Koroteev \\ International Laser Center, M. V. Lomonosov Moscow State University \\ Vorob'evy Gory, Moscow 119899, Russia
}

\begin{abstract}
An experience of managing the continuing education and retraining programs at the International Laser Center (ILC) of Moscow State University is discussed. The offered programs are in a wide range of areas, namely laser physics and technology, laser biophysics and biomedicine, laser chemistry, and computers in laser physics. The attendees who are presumably scientists, engineers, technical managers, and graduate students can join these programs through the annual ILC term (6 months), individual training and research programs (up to a year), annual ILC Laser Graduate School, graduate study, and post-docs program, which are reviewed in the paper. A curriculum that includes basic and specialized courses is described in detail. A brief description of the ILC Laser Teaching and Computer Labs that support all the educational courses is given as well.
\end{abstract}

\title{
1. WHAT IS THE ILC MSU?
}

International Laser Center of Moscow State University (ILC MSU) established in 1989 is a chapter of the ILC of Eastern and Central European countries at Moscow State University having its own teaching, research, and supporting staff, budget, and property. ILC MSU's activities involve also scientific and teaching staff of the Departments of Physics, Chemistry, Biology, and Research Institute of Physico-Chemical Biology named after A. N. Belozersky. ILC MSU is a coordinating organization of the ILC of Eastern and Central European countries. The main purpose of the ILC MSU is to govern all the activities in laser physics within the MSU, to offer scientists the necessary tools for the laser research, and to provide the continuing education and international cooperation programs.

The ILC MSU key activities fall into three major parts: 0pt4pt plus 2pt minus 1pt

- Fundamental research program

- Development of unique laser equipment

- Continuing education and retraining program

Here, we will be focused mostly on the ILC MSU continuing education and retraining program.

\section{ILC CONTINUING EDUCATION AND RETRAINING PROGRAM}

ILC MSU offers for the scientists and specialists from Universities, Russian Academy of Sciences, and industry the continuing education and retraining program in the following range of areas: $0 \mathrm{pt} 4 \mathrm{pt}$ plus $2 \mathrm{pt}$ minus $1 \mathrm{pt}$

- Laser physics and technology

- Laser biophysics and biomedicine

- Laser chemistry

- Computers in laser physics.

The attendees can join these programs through the annual ILC term (6 months), individual training and research programs based on the specific curriculum for the trainee (from 2 to 12 months), annual ILC Laser Graduate School, graduate study, and post-docs program. 


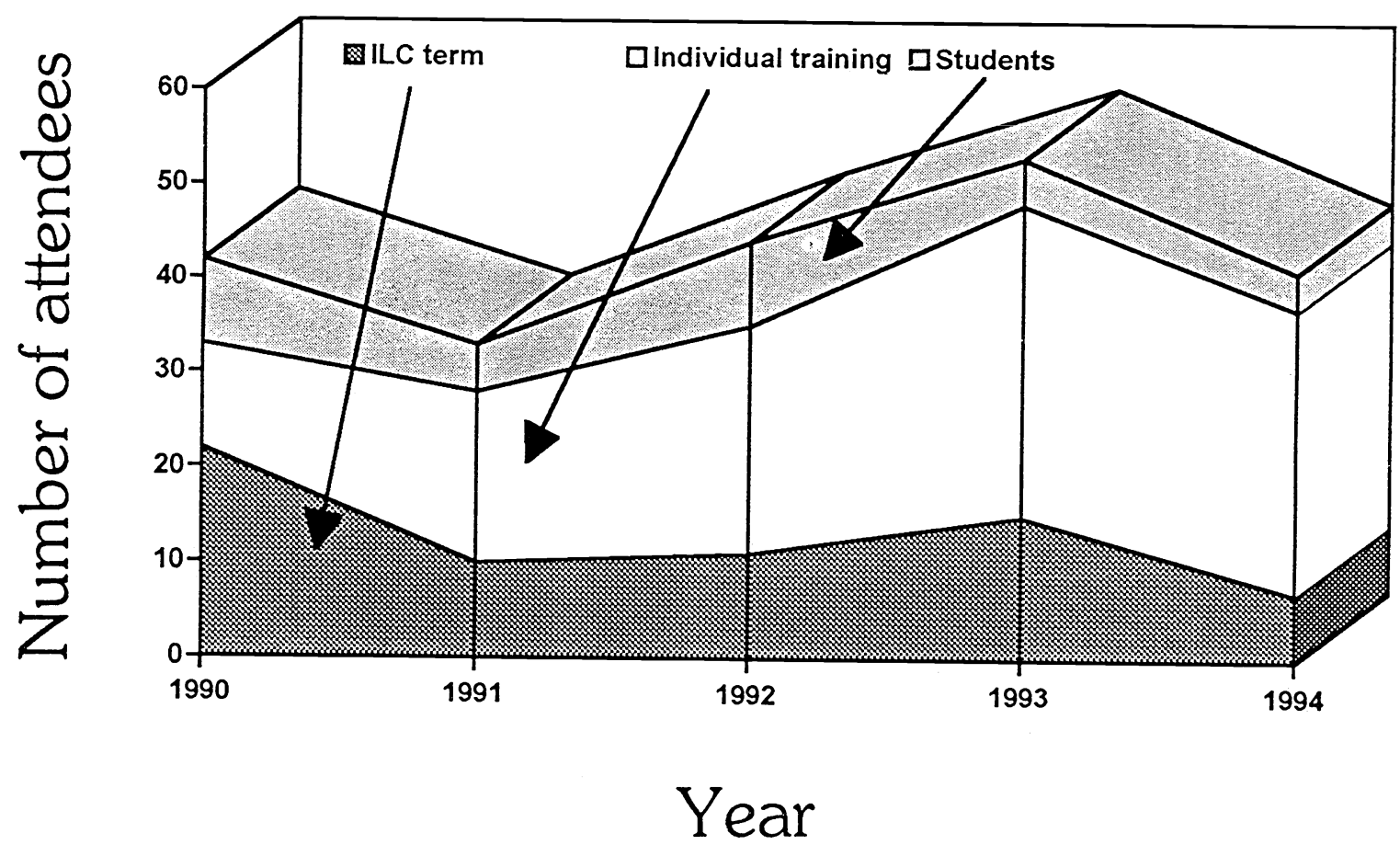

Figure 1: ILC continuing education and retraining activities in numbers.

\subsection{Annual ILC Term}

The annual ILC term is a key activity that starts annually in January and lasts for 6 months. The curriculum includes a fundamental training that is provided through the basic courses ("Computer-controlled data acquisition systems" (Sec. 3), "Experimental laser physics" (Sec. 4), "Theoretical Fundamentals of Quantum Electronics" (Sec. 5), and "Matter in a strong laser field" (Sec. 5), which are obligatory and followed by exams for all the trainees. The basic courses provide fundamental knowledge in Laser Physics and are based not only on theoretical cognition, but also on training in the ILC MSU Laser Teaching Lab (Sec. 4.2) and in the Computer Lab (Sec. 3.1). All the courses are supported by specially written and edited notes and textbooks.

The curriculum includes also an individual training that is provided by a great variety (more than 30 ) of specialized courses (Sec. 6) offered to the trainees for choice (at the ILC term the trainee should take some 5 specialized courses). Specialized courses are designed to extend the general courses program.

In addition, the trainees should spend up to $50 \%$ of the training time in the ILC MSU's labs (we also widely use for this purposes the laboratories of the Russian Academy of Sciences) to carry out specific research projects.

In the end of the ILC term a diploma is granted on the basis of the scientific research and public presentation of the completed investigation.

\subsection{Individual Training and Research Programs}

In addition to the ILC term, we offer the attendees an individual training and research programs based on the individual curriculum. In this case, the trainee spends regularly most of his/her time in the laboratory, making research, and study just several specialized courses on the scientific advisor's advise. No diploma is granted in this case.

The individual training and research programs are the most suitable choice for those who previously attended the ILC term and are going to continue the research, for graduate students and post-docs. After the ILC term every year, $20-30 \%$ of the attendees joint this individual programs and, finally, $2-3$ of them became the graduate students of the ILC MSU. 


\subsection{Continuing Education and Training Activities in Numbers}

Fig. 1 shows the dynamics of the total number of trainees attending both the ILC term and individual programs for the period of 1990-1994. In this dynamics there is a clear tendency of increasing the number of attendees who would like to have an individual training. At the same time, the number of students and people attending the ILC term is about the same for the period of 1991-1994.

\subsection{Other Educational Activities}

ILC MSU organizes, since 1990, on the annual basis the ILC Laser Graduate School (Sec. 7). It consists of the set of short (2-8 hours) courses delivered in English by key scientists from all over the world. The aim of the LGS is to bring the audience into the focus of up-to-date research in the specific area.

In addition, ILC MSU has a special department of English for Russian speaking students and Russian for foreigners (Sec. 8).

\section{BASIC COURSE "COMPUTER-CONTROLLED DATA ACQUISITION SYSTEMS"}

This basic course is intended to answer the following questions: 0pt4pt plus 2pt minus 1pt

- How to design and construct a computer-controlled data acquisition system for your experimental set-up?

- How to register/generate the experimental data?

- How to analyze the experimental data? Which software packages can you use for that?

The course (delivered by Dr. Yu. V. Ponomarev, Dr. V. N. Zadkov, Dr. N. N. Kurochkin) consists of the lectures (32 hours), computer labs (16 hours), and practicum (24 hours). A basic programming language for this course can be wether "C" or "Pascal" (we use "Turbo"-compiler versions). The program of lecture course is given below. 0pt4pt plus 2pt minus 1pt

1. PC-controlled experimental data acquisition systems: Hardware and software architecture and design.

2. How to get/register the experimental data? Practicum examples. Generation of experimental-like data on a computer.

3. Experimental data analysis methods: Statistical, optimal filtration, correlation and spectral analysis.

4. Software packages for modeling and analyzing the experimental data: Libraries of "C" and "Pascal" routines, general and specialized packages, scientific computer graphics.

The attendees of this course gain their practical skills in the computer labs and practicum. After several teaching computer labs, every student has to do a specific computer project on design the software package for the PC-controlled data acquisition system of a real experiment he/she will do then in practicum. In the computer lab the experimental set-up is substituted by special routines that generate the experimental-like data.

After that, the attendees move on to the practicum, where they should make first several teaching projects and, finally, continue with their projects they started in the computer lab.

Practicum consists of a set of teaching projects based on IBM-like computers, CAMAC or ISA-based interfaces, different hardware to register/generate signals, etc. All the projects are based on the real physical set-ups. In addition, we widely use the analog computers to model a physical phenomena (for instance, chaos or strange attractor) first and then a PC uses to register the experimental data, to analyze them and present graphically.

In the end of the course, the attendees have to present the results of their projects in a report-like form.

\subsection{ILC Computer Laboratory}

The ILC MSU computer facilities can be divided into two major groups. The first group consists of a plenty of PCs, which are scattered in the experimental and theoretical labs. The second group includes all the equipment that is in common use and is located at the ILC Computer Lab, namely, 2 DEC AXP 3000-300X workstations (based on the 64-bit ALPHA chip), VAX 4000-200 microcomputer with several VTs and DEC PC433 workstation, and 25 i386 and i486-based PCs served by a LAN server. Most of the PCs around the ILC are connected to the ILC local network 
(Ethernet-based). In addition to the print/file server facilities, an Internet link is available, providing a wide access to the e-mail, FTP, telnet, etc. All the common-in-use computer facilities are used both for educational training and scientific research.

The ILC MSU is open for any cooperation with leading computer companies. For example, two years ago a Collaborative Engineering Center between Digital Equipment Corporation and M.V.Lomonosov Moscow State University was established at the ILC MSU. This center is a linking body between MSU and DEC and its aim is to apply a scientific potential of MSU scientists and the newest DEC technologies to a great variety of applications, especially to software development.

\section{BASIC COURSE "EXPERIMENTAL LASER PHYSICS"}

The purpose of this course is to introduce for the attendees some key theoretical and experimental knowledge on laser physics and nonlinear optics that is necessary to work then in an experimental laboratory. The course consists of the lectures/seminars (16 hours) and practicum (42 hours).

\subsection{Theoretical Introductory Course (Dr. B. V. Zhdanov)}

The short theoretical introductory course that is not a substitute for the regular theoretical courses on quantum electronics and nonlinear optics is focused on the key theoretical issues and practical tips, which are necessary for the attendees to make successfully all the experimental teaching projects in the practicum. The program of this course is listed below.

Introduction. Structure of the practicum and the ILC Laser Teaching Lab. Teaching projects: How to do them? Safety rules in a laser laboratory.

Generation of ultrashort laser pulses. The necessary conditions to synchronize the longitudinal and transverse modes in a passive resonator. Coherence and spectral limitations. How to synchronize the modes in an active resonator? Amplitude modulation of gain or losses. Different techniques of active and passive mode synchronization. Propagation of the ultrashort laser pulses in a real laser's resonator. Group velocity.

Optics of anisotropic media. A tensor. Transformation of tensor's components. 2nd and 3rd rank tensors. Crystals and symmetry. Laboratory and crystallographic frames. How to use tensors to describe the crystal's symmetry properties? Light propagation in an anisotropic media. Linear electrooptical effect. Sum- and difference-frequency generation.

Fundamentals of nonlinear optics. A subject of nonlinear optics. Nonlinear polarization of nonlinear media. Examples: nonlinear oscillator, gas of electrons. Classification of the effects and equations of nonlinear optics. Averaging over oscillations. Role of dispersion in nonlinear optics. Phase-matching for nonlinear wave interactions. Generation of optical harmonics. Second harmonic generation (SHG). A length of coherent interaction. Optimal beam focusing. Intracavity SHG. Generation of higher harmonics. Optical parametric amplification and generation. Optical parametric oscillator (OPO): its characteristics. Parametric luminescence. Nonlinear optical effects in a media with cubic nonlinearity. Self-action of light waves. Self-focusing of the light beams. Resonance nonlinear effects. Stimulated Raman scattering. Nonlinear spectroscopy. Four-wave mixing spectroscopy. Coherent anti-Stokes Raman spectroscopy (CARS).

Techniques for measure the characteristics of laser radiation. Main parameters of laser radiation: energetic, spectral, temporal, spatial. Detectors: their theoretical description, different operation regimes. Heat-sensitive detectors. Photoeffect: photoelement, photomultiplier, microchannel detectors, electro-optical convertor; photoresist, detectors with p-n transition. Noise: sources and characteristics. Detector's characteristics. Techniques of detecting the single photons: synchro-detection, photon counting. Statistical analysis of experimental data.

\subsection{Practicum and the ILC Laser Teaching Lab}

In the practicum the attendees get the knowledge of the basic principles of laser operation and nonlinear optics by making first model experiments with analog and digital computers. Then, they make real physical experiments in the ILC Laser Teaching Lab using laser teaching set-ups, which look and act like real physical experimental set-ups. While in the teaching lab, the attendees study how to perform some basic experimental operations-align optical elements and systems, tune devices, use different techniques of conducting the experiments, methods of experimental 
data acquisition, etc., and gain therefore finally the experimental skills which are necessary to conduct the experiments in scientific labs.

The ILC Laser Teaching Lab offers the attendees 9 basic experimental projects to work on. They are described below.

Nd:YAG Laser Radiation Kinetics. The purpose of this experimental project is to acquaint attendees with a solid-state laser construction, principles of its operation, and basic tools to measure the laser radiation parameters. A teaching set-up (Fig. 2) contains a cw-pumped Nd:YAG laser with an acousto-optical Q-switch, He-Ne laser for the alignment of the Nd:YAG laser cavity, power meter, frequency doubler, photodetector, oscilloscope, and spectroanalyzer.

In this project, two laser operating regimes are to be investigated, namely $\mathrm{cw}$ and $\mathrm{Q}$-switched regimes. The attendees first should adjust the laser cavity elements to receive a generation of laser radiation and then measure the laser generation threshold. After that, they should observe the relaxation oscillations in the intensity of laser radiation, measure their frequency and dependence on the pump power. For the Q-switched regime, the giant pulses parameters have to be measured (pulse duration, peak power, and its dependence on repetition rate).

Finally, the attendees should obtain a second harmonic generation (SHG) in $\mathrm{LiNbO}_{3}$ crystal for the both laser generation regimes and observe a sharp increase in the SHG signal while Q-modulation is switched on.

Ultrashort Light Pulses: Generation and Measurement Techniques. This experimental project meets the attendees with the generation of ultrashort laser pulses produced by a cw-pumped Nd:YAG laser with acousto-optical modulation and mode-locking and pulse duration measurements based on the correlation technique.

The set-up (Fig. 3) consists of a mode-locked and Q-switched Nd:YAG laser (homemade at the ILC MSU), fast photodiode and an oscilloscope to register the temporal dependences of laser radiation intensity, power meter, frequency doubler, and a computer-controlled optical correlator to measure the pulse duration.

Here, the attendees investigate all possible laser operation regimes, such as $\mathrm{cw}, \mathrm{Q}$-switched, active mode-locking, Q-switched and mode-locking simultaneously. They measure an average radiation power for the Q-switched regime, and laser pulse duration.

An important issue of this project, plus to the study the ultrashort laser pulses generation techniques, is that the attendees study also an optical correlation technique to measure the ultrashort optical pulses duration using a computer-controlled correlator based on a Michelson-interferometer-type scheme.

Optical Harmonics Generation. Two key nonlinear effects such as second harmonic generation and optical mixing are studied experimentally in this project.

The set-up consist of a pulsed Q-switched Nd:YAG laser, two special positioners for nonlinear crystals, power meter and three photodiodes for intensity measurements, and a computer- controlled data acquisition system.

In the first section of the project, the main factors which determine the SHG efficiency are studied. Attendees first investigate a role of phase-matching conditions, then a dependence of SHG efficiency on laser radiation power for different nonlinear crystals $\left(\mathrm{KDP}, \mathrm{LiNbO}_{3}, \mathrm{LiO}_{3}\right)$. By the end of the section, the attendees should calculate the relative nonlinearities of these crystals using the experimental data.

In the second section of this project, the generation of third and fourth optical harmonics is studied using a two-cascade generator.

Optical Parametric Oscillator. This teaching project is focused on one of the basic nonlinear optical phenomena -optical parametric generation and introduces for the attendees two nonlinear optical devices: an optical frequency doubler and an optical parametric oscillator (OPO), which is a source of tunable in a wide spectral range coherent optical radiation.

The set-up (Fig. 4) consists of a pulsed Q-switched Nd:YAG laser, two special devices for the frequency doubling and for the OPO cavity, power meter, IR to visible convertor for observing the parametric generation, monochromator, photodiode, and an oscilloscope to measure the wavelength of parametric generation.

First, the attendees tune the optical frequency doubler and measure the intensity of the SHG, which is used to pump the OPO. Then, the OPO resonator should be align. The parametric generation can be observed on the converter's screen. The attendees measure the OPO generation threshold. After that they measure the dependence of radiation wavelength on the angle between the laser beam and the crystal axis-an OPO tuning curve.

Optical Modulators. This project is centered on the basic principles of modulation of optical radiation. Here, the attendees study two types of optical modulators: the electro-optical and acousto-optical ones.

The set-up consists of a He-Ne laser, electro-optical modulator, acousto-optical modulator with a high frequency generator, photodetector, oscilloscope, mirrors, lenses, and polarizer. 


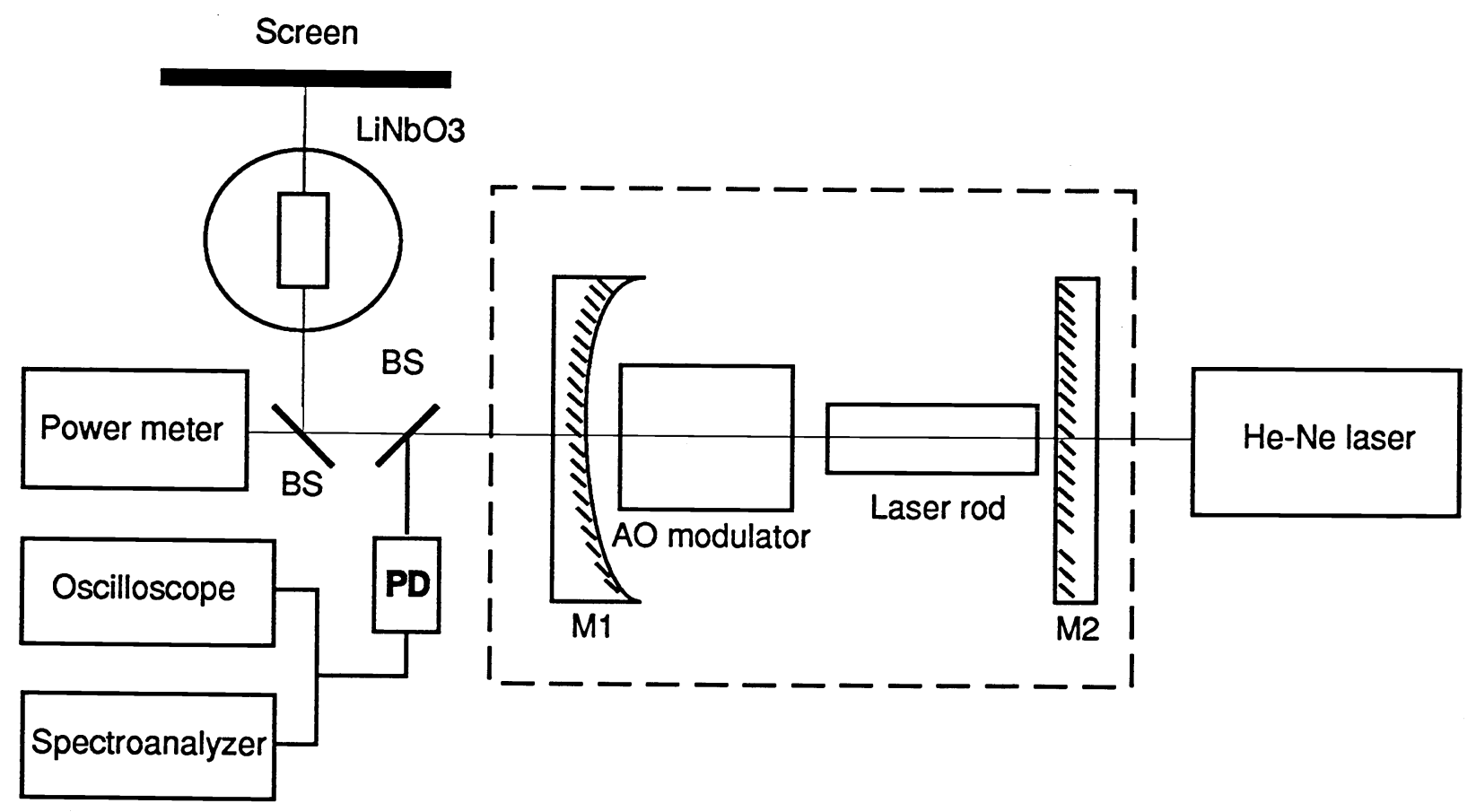

Figure 2: Teaching set-up for the experimental project on Nd:YAG laser radiation kinetics.

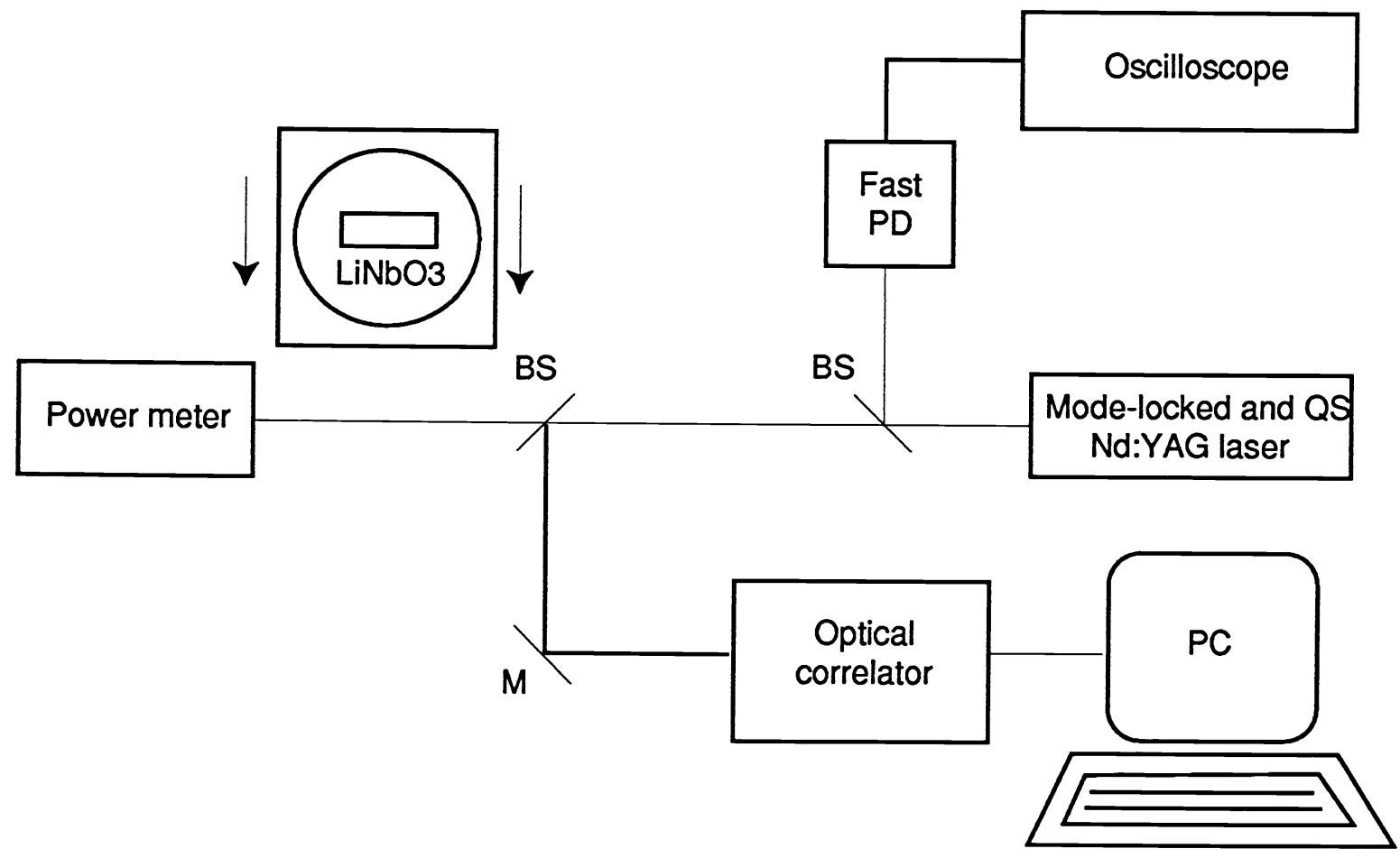

Figure 3: Teaching set-up for the experimental project on ultrashort light pulses. 
First, the attendees study an electro-optical modulator. They align the modulator and observe a conoscopic pattern and its transformation due to supplied voltage variations. Then, they measure the dependence of transparency of the Pokkels cell on the electric field strength and the half-wave voltage of this cell. Using these data they calculate the value of the electrooptical constants.

After that, the acousto-optical modulator (AOM) is studied. The attendees adjust the vertical and horizontal positions of the rotatable stage supporting AOM. After turning on the HF generator connecting to a piezoelectric transducer of AOM they can observe the diffraction pattern on the screen, measure the Bragg diffraction angle and its dependence on the acoustic wave frequency.

Finally, the attendees measure the phase-matching angle width and the dependence of the diffracted light beam efficiency on the acoustic wave power. Using the experimental data they can calculate also the sound speed in quartz.

Holography. This project meets the attendees with a practical technique of recording the holograms from a real transparent object. To study this technique, the attendees use a kit containing a He-Ne laser, optical mirrors, beam splitter, magnet-based mounts and holders, and different transparent objects.

First, the attendees select a scheme of experimental set-up, calculate the position of the object corresponding to the optical elements. Then, they design the set-up and align optical elements to obtain two coherent beams (so called "object" and "reference" beams). These two beams are used to record the hologram on a photoplate. After the developing the photoplate, the attendees install it into the set-up and illuminate by the reference beam. As a result, they can see a virtual holographic image of the object and the virtual or real image-doubling.

CARS Spectrometer. This project is concerned with a nonlinear spectroscopy technique-Coherent Anti-Stokes Raman Spectroscopy (CARS), and is very important by means of gaining the experimental skills on carrying out a modern laser experiment.

The spectrometer (Fig. 5) consists of a pulsed passive Q-switched Nd:YAG laser, an amplifier, KTP frequency doubler and a broad-band dye laser. In CARS experiment the attendees use the second harmonic of the laser frequency $(0.5321 \mu \mathrm{m})$ and the radiation of the dye laser $(0.55-0.66 \mu \mathrm{m})$. The two beams are focused in a sample. In this project the attendees investigate two-isotope gas mixture of $\mathrm{CO}_{2}$ molecule. The anti-Stokes radiation after passing through the polychromator is registered by an Optical Multichannel Analyzer (OMA). It allows, in combination with the polychromator, to observe and register the whole spectrum of anti-Stokes radiation during a laser pulse. A PC-based data acquisition system is used to control over the experimental set-up.

The attendees starts this project with the operating of OMA. They observe the spectrum of the SHG and dye laser radiation, measure the spectral resolution of OMA and the spectral width of dye laser radiation. Then, after tuning the dye laser frequency to the resonance of a sample, they observe the CARS-spectrum of the sample $\left(\mathrm{CO}_{2}\right.$ under the high pressure). After that the attendees measure the CARS-spectrum of the isotopes in molecular gas mixture, the Raman shifts and the relative concentration of each isotope.

Additionally, the advanced attendees can carry out their own scientific research with a new sample.

Adaptive Systems. The purpose of this project is to teach the attendees the operation principles of adaptive systems.

The set-up consists of a pulsed Nd:YAG laser with the computer-controlled cavity mirrors, TV camera and a monitor for observing the laser beam intensity profile, and a PC-based data acquisition system.

Here, attendees study the adaptive system, which can control two cavity mirrors and therefore optimize the laser radiation parameters. As a result, this system can maximize the radiation intensity along the laser beam, when the thermal distortions in the laser rod take place. The attendees watching for these operations on the TV screen can see how the aberrations in the laser rod can influence on the intensity profile of the laser beam and how this influence can be compensated due to the changes in the mirrors' shapes.

Laser Doppler Anemometer. In this project the attendees meet with the basic principles of laser Doppler anemometry. The set-up consists of a He-Ne laser, telescope, rotating light scattering disc, two lenses, photodiode, oscilloscope, and a PC-based data-acquisition system.

The Doppler anemometry method is used here to measure a velocity of the rotating light scattering disc. The attendees align the optical elements to obtain and optimize the Doppler signal. Then, they measure the velocities of the different points of the rotating disc. The signal from the photodiode is analyzed on the computer using the fast Fourier transfom routine. 


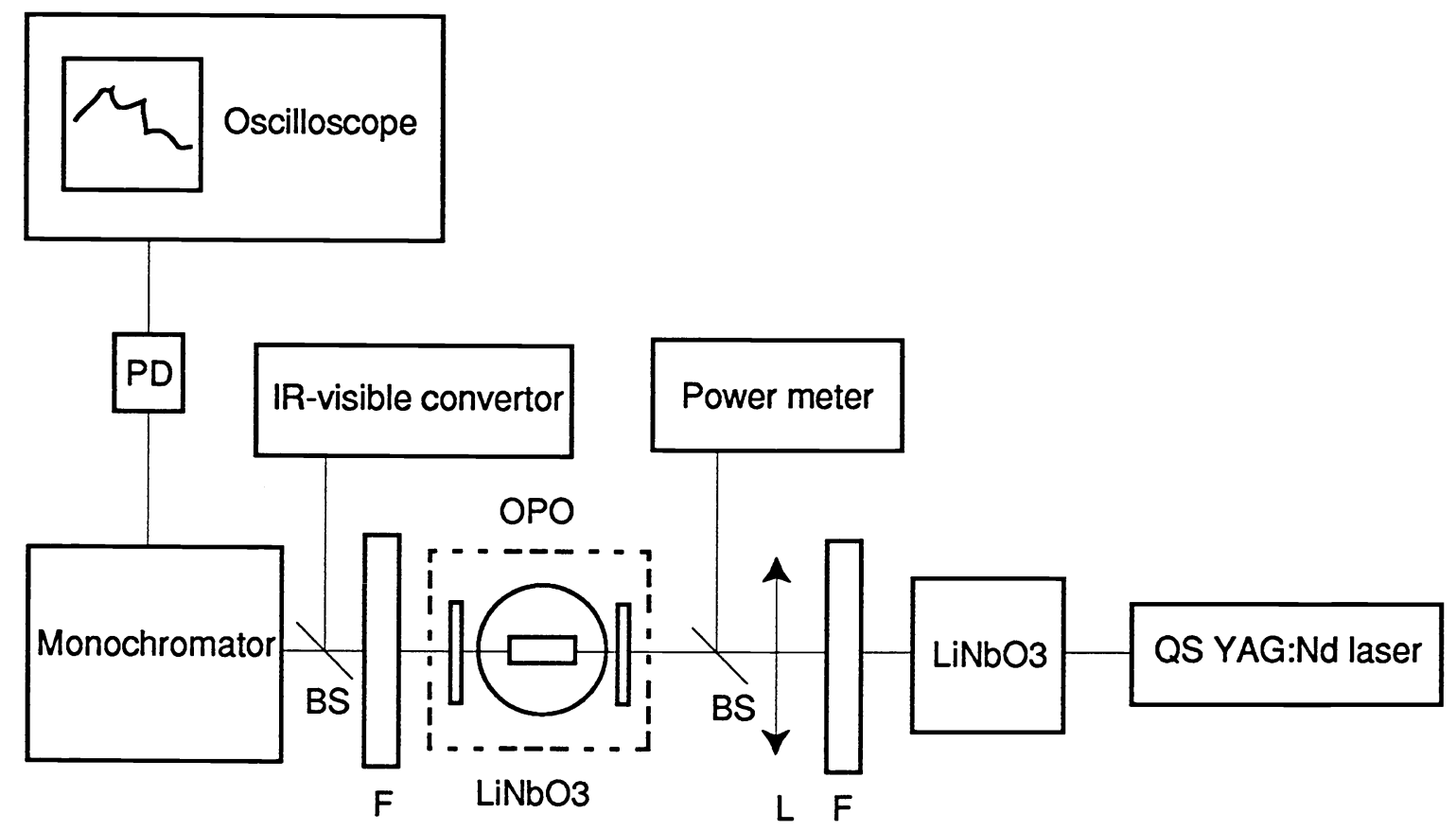

Figure 4: Teaching set-up for the experimental project on optical parametric oscillator.

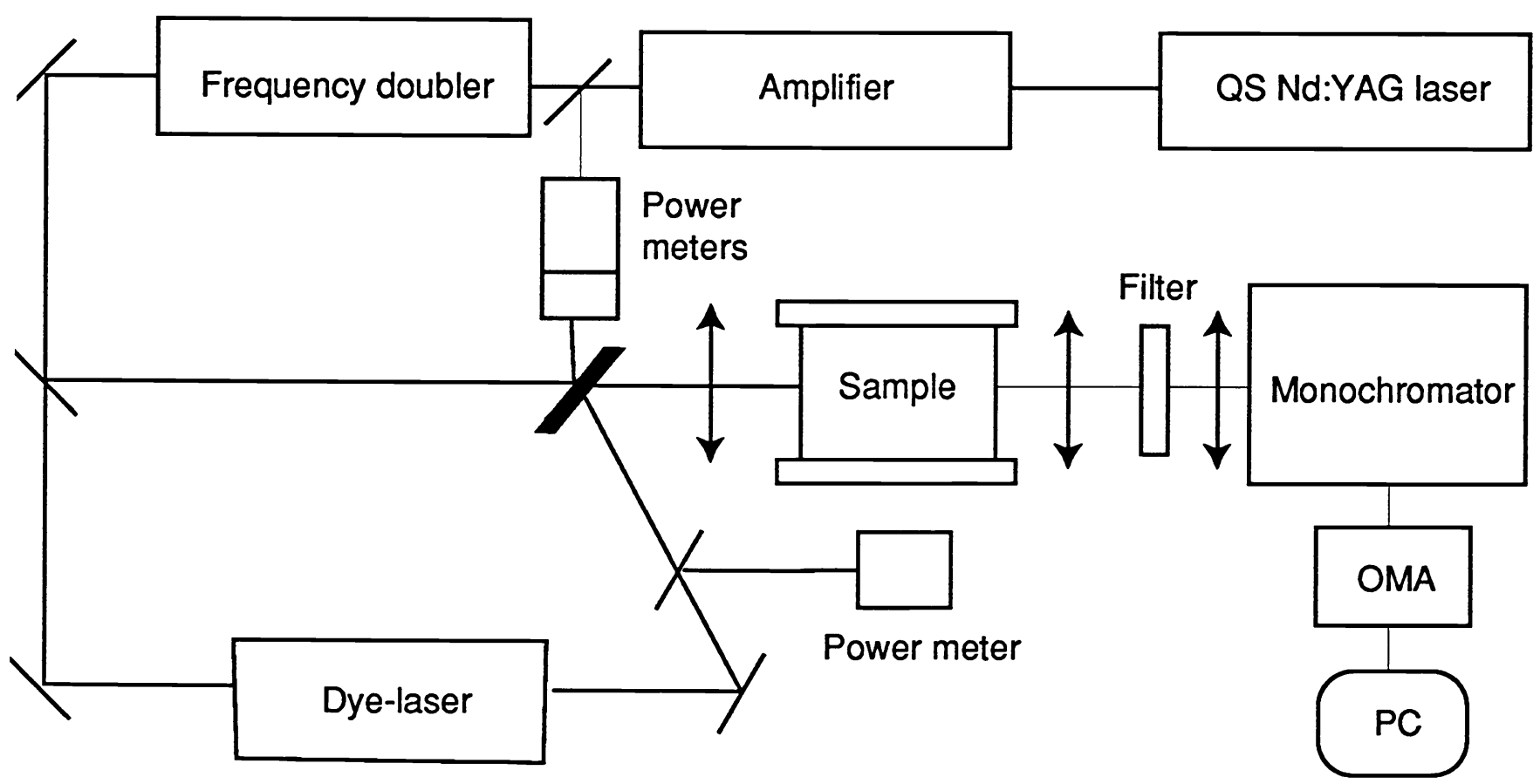

Figure 5: Teaching set-up for the experimental project on CARS-spectrometer. 


\section{OTHER BASIC COURSES}

\subsection{Theoretical Fundamentals of Quantum Electronics (Prof. V. I. Emel'yanov)}

This lecture course (32 hours) is an introduction to the basic notions and theoretical methods of quantum electronics and is intended to the attendees with a minimum knowledge of quantum mechanics.

The aim of the course is, first of all, to facilitate grasping of the intuitive understanding of physical effects in quantum electronics, to learn how qualitatively analyze and semiquantitatively evaluate them. The peculiarity of this course consists in that the broad class of the basic effects of quantum electronics is considered on the basis of a unified simple model of ensemble of quantum two-level systems, interacting with classical electromagnetic and lattice displacement fields.

Starting with the Shrödinger equation in the energy representation, the model of two-level approximation with allowance for the relaxation terms is obtained. The problems of the interaction of immobile atoms with the given electromagnetic field are considered: light absorption and amplification, field broadening and saturation effect are among them. The qualitative difference in the behaviors of dissipative and conservative systems is discussed on the example of the nutation effect. The problem of light absorption and amplification by the ensemble of chaotically moving atoms in gas illustrates the role of inhomogeneous line broadening. On the basis of the same model, the problem of laser generation in a resonator is considered. The role of dissipation, stimulated emission, and saturation effect is cleared out. The qualitative difference between the process of stimulated emission of photons and the process of collective spontaneous emission (superradiance) is discussed. The basic elements of the solid state theory, the electronic band energy spectrum, and optical and acoustical lattice vibrations are considered. Using the model of the two-level continuum the light absorption and amplification in semiconductors are considered.

In the frame of the unified model, the semiquantitative microscopic consideration of spontaneous and stimulated Raman scattering of light on optical phonons and stimulated Brillouin scattering are studied. The nonlinear optical susceptibilities of the model are introduced, the classification and the discussion of the nonlinear optical processes are carried out, in particular the parametric wave interactions, harmonics generation, the frequency up-conversion, optical rectification. The concluding lecture of the course is dedicated to the review of the modern trends in quantum electronics.

\subsection{Matter in a Strong Laser Field (Prof. N. I. Koroteev)}

The subject of this course is a matter in a strong laser field. Concentration of energy in optical pulses produced by the most powerful laser systems far exceeds energy concentration in matter in the form of chemical bonds' energy and is somewhat lower than a concentration of nuclear energy. Concentration of the momentum in such laser beams is also enormous. Then, the question arises as to whether it is possible to transfer laser energy to matter and through what stages? In what way structure of matter and its inhomogeneity may influence laser-matter interaction? The course provides the answers to these and many other related questions.

First, laser interaction with the free charges and plasmas is analyzed in detail. The inverse bremsstrahlung effect, resonance absorption of laser radiation in plasma are considered. Other topics include the problem of laser fusion, light pressure effects, optical breakdown of transparent dielectrics, behavior of atoms, molecules, and condensed matter in a strong laser field. Special attention is paid on the role of interfaces in the surface-light-field interaction.

\section{SPECIALIZED COURSES}

In addition to the set of basic courses that are obligatory for the ILC attendees, ILC offers more than 30 specialized courses for choice. A selected list of them is given below.

Laser Physics: "Laser physics", "Physics and techniques of gas lasers", "Optics of ultrashort light pulses", "LIDARs and laser spectroscopy of the environment", "Intense laser fields: Physics and methods of generation", "Nonlinear optics", "Physics of fiber-optical devices", "Adaptive optical systems", etc.

Laser biology and biomedicine: "Lasers in optical microscopy", "Laser diagnostics in biology and medicine", "Cellular laser surgery", "Lasers in biological investigations: Problems, achievements, and perspectives", etc.

Fundamentals of laser technology: "Physical fundamentals of laser technology", "Laser optics and thermal physics of surface", "Laser-induced modification of material properties", etc.

Computers in laser physics: "Optical information processing", "Computational physics", etc. 


\section{ILC LASER GRADUATE SCHOOL}

How to bring the students into the focus of the up-to-date scientific and technological advantages in laser physics and in related fields? No one from the regular basic or specialized courses can cover this gap. In addition, these courses last for 2-3 months that is sometime not suitable for the specialists who would like to get only very fresh, new information, or they would like to move on to another field of their scientific research and they have no enough time to attend the ILC term or even to attend some selected courses.

In this case, the most suitable educational activity ILC MSU could offer them is the ILC Laser Graduate School (ILC LGS) - session that lasts for 1-2 weeks and consists of short courses (2-8 hours each) delivered by the instructors who are the top scientists in the specific research through all over the world. Each course is focused on a selected scientific subject and covers the introduction to the discussed field, some history, major and up-to-date results, new perspectives, and key references. Thus, a short course is basically an overview of the selected subject given by a top specialist in this field.

Short courses are quite popular among the scientists abroad and their sessions follow regularly any large conference organized by OSA or SPIE, especially in the US. During last decade, the short courses sessions became a regular educational activity at many laser centers and universities in the US. They take place several times a year and attract mostly people from industry, medicine, military bodies.

In Russia, the short courses program activity has (plus to all the other advantages) thanks to the financial problems we have now an extra value being now the only opportunity for the students (graduate students, young scientists, etc.) to "touch" the Science not just reading the papers in scientific journals, but attending the instructor's courses, talking to them, asking questions.

All the above mentioned reasons led to the organizing on the annual basis the ILC LGS that was initiated in 1990 by the late Prof. Sergei Akhmanov and has by the moment a 6-years history (Fig. 6). Started with the first LGS'90, Workshop on "Modern Problems in Laser Physics" that consisted of the short courses in different fields of laser physics, laser biology and biomedicine, and computer physics, we came in 1992 to the idea of focusing the every session or the Workshop of the LGS on a selected, quite narrow field. This helped us a lot in attracting the best instructors that could cover the subject of the Workshop completely. Additionally, the "specialization" led to the increase in the number of the LGS attendees, and, finally, the ILC LGS became not only an educational event, but a scientific one as well.

- 1990 Workshop on Modern Problems of Laser Physics; Workshop on Laser Biophysics

- 1991 Workshop on Modern Problems of Laser Physics

- 1992 Workshop on Modern Problems of Laser Physics; Workshop on Photoacoustics

- 1993 Workshop on Nonresonant Interaction of Laser Radiation with Matter

- 1994 Workshop on Surfaces and Interfaces: Laser Induced Processes and Nonlinear Optical Diagnostics

- 1995 Workshop on Imaging Through the Atmosphere: Wave Propagation and Adaptive/Nonlinear Optics Techniques

Figure 6: ILC Laser Graduate School: Milestones.

By now, the ILC LGS has a good reputation and is a well-known meeting within not only Moscow and Russia, but the FSU states as well due to the high-level of the instructors we invite for the Workshops. Every year we try to invite an international team of top scientists in their fields. Among the ILC LGS instructors were H.Walther (FRG), C.Flytzanis (France), A.Boccara (France), D.Furnier (France), A.Newell (USA), A.Laaksonen (Sweden), C.Rulliere (France), H.Coufal (USA), G.Diebold (USA), G.Kino (USA), R.Thomas (USA), L.Favro (USA), A.Mandelis (Canada), J.Murphy (USA), A.Tam (USA), P.Hess (FRG), H. van Driel (Canada), T.F.Heinz (USA), T.Furtak (USA), and many others. An example of the LGS'94 scientific program, Workshop on "Surfaces and Interfaces: Laser Induced Processes and Nonlinear Optical Diagnostics", is given in Fig. 7.

The attendees of the ILC LGS are the students of Physics (4th, 5th, and 6th-year study), graduate students, scientists from the Universities and Institutes of the Russian Academy of Sciences, people from industry from Moscow 


\title{
Laser Graduate School \\ Workshop on Surfaces and Interfaces: Laser Induced Processes and Nonlinear Optical Diagnostics
}

\author{
Moscow, Russia, April 11-15, 1994
}

\section{Organized by}

International Laser Center, Moscow State University

In co-operation with

Scientific and Research Center for Technological Lasers, Russian Academy of Sciences

\section{Short Courses List}

\section{SC1 4 hours, April 11, 1994}

Henry Van Driel, University of Toronto, Canada

"Nonlinear Optics at Nonideal Semiconductor and Metal Surfaces"

SC2 4 hours, April 11, 1994

Tony F. Heinz, IBM T.J.Watson Research Ctr, Yorktown Heights, NY, USA

"Surface Science on the Femtosecond Time Scale"

SC3 4 hours, April 12, 1994

Thomas E. Furtak, Colorado School of Mines, USA

"Surface Enhanced Nonlinear Optical Phenomena"

\section{SC4 4 hours, April 12, 1994}

Theo Rasing, University of Nijmegen, The Netherlands

"Nonlinear Optical Spectroscopy at Surfaces and Interfaces"

SC5 4 hours, April 14, 1994

Ole Keller, University of Aalborg, Danmark

"Near-Field Optics: On the Microscopic Theories and the Applications in Basic Physics"

SC6 4 hours, April 14, 1994

Dietrich von der Linde, Universitat Essen, Germany

"Ultrafast Solid-Liquid Phase Transformations"

SC7 4 hours, April 15, 1994

Enno Arenholz, J.-Kepler University Linz, Austria

"UV-Laser Induced Surface Modifications of Organic Polymers"

SC8 2 hours, April 15, 1994

Vladimir Emel'yanov, Moscow State University, Russia

"Laser-Induced Periodic Defect and Damage Structures on Surfaces and Films"

SC9 2 hours, April 15, 1994

Oleg Aletsipetrov, Moscow State University, Russia

"Harmonic Generation and Nonlinear Optical Diagnostics of Surfaces"

Poster Session, April 15, 1994

All the attendees who like to make poster presentations are welcome to the Poster Session

Figure 7: The scientific program of the ILC LGS'94. 
and Moscow region, other places in Russia and the FSU states. We had also some experience in hosting attendees from Check and Slovak republics, Bulgaria, Belgium, and Norway. This year, at the LGS'95, Workshop on "Imaging Through the Atmosphere: Wave Propagation and Adaptive/Nonlinear Optics Techniques", the total number of attendees was 280 starting from 80 at the LGS'90 in 1990.

All the courses at the ILC LGS, questions, and discussions are delivered in English. This, in spite of some difficulties for those students who are not good in English (Sec. 8), creates a special atmosphere of the School close to that one of the regular scientific meetings and therefore helps students in raising an experience in scientific communications. For the advanced students we offer during a Workshop a poster session, which is a room for them to present their own scientific results.

\section{ILC LINGUISTICS LABORATORY}

There is no doubt that for scientists an ability to communicate easily with their colleagues orally (at the international meetings) or in a written form (writing papers, letters, via e-mail) is an extremely essential component of their education. Now, English is a foreign language, which scientists mostly use for communication purposes. For a nonEnglish-speaking country like Russia a good knowledge of English, especially as a tool for communication purposes in Science, could be (and is) a great problem for the majority of scientists and engineers. This is why shortly after establishing the ILC MSU a special ILC department-the ILC Linguistics Lab-has been created. Its aim is to give the ILC students and attendees the necessary skills of English for scientific and every-day use and therefore help them in creating and extending their international contacts and cooperation.

Classes of English are focused mainly on the oral language skills and those tools, which are necessary to deliver an oral paper at a meeting or to make an oral presentation, to participate at a seminar or a discussion, for personal informal discussions, etc. A special attention is paid here on the listening comprehension skills. Some classes are conducted by native-speakers of English who offer an additional information on the country they came from. Many other classes are also based on the American or British history, culture, customs and use the newspapers and books, movies, records and TV-programs. All this help the attendees a lot when they are going to participate in a conference abroad.

In addition, several specialized courses on "Oral Scientific Discourse", "Business Writing", etc., are offered for the attendees. Plus, the laboratory stuff helps in preparing the necessary materials for the conferences, proceedings, and collection of papers.

The 5-years experience in teaching English for the ILC attendees led finally to a project of a special English course for the students of Optics. Its idea is to teach English based on the Optics and Laser Physics courses. This project is now under way.

\section{ACKNOWLEDGEMENTS}

A great help of Dr. B. V. Zhdanov, Dr. Yu. V. Ponomarev, Dr. M. N. Kozyreva, Dr. I. V. Fedorova, and Mrs. L. I. Pentegova in preparing the materials for this paper is highly appreciated. 